\title{
Evaluación del proceso de decanulación en pacientes traqueostomizados en el Hospital Carlos van Buren. Una cohorte retrospectiva
}

\section{Evaluation of decannulation process in tracheotomized patients at Carlos van Buren Hospital. A retrospective cohort}

\author{
Cristian Cortés $\mathbf{Q}^{1}$, $\mathbf{M}^{\mathrm{a}}$ José Gálvez $\mathrm{M}^{2}$, Franco Moya $\mathrm{D}^{3}$, Dominique Perrot $\mathrm{T}^{4}$, Paula Guerra $\mathbf{R}^{5}$, \\ Cristian Papuzinski $A^{6}$.
}

\begin{abstract}
RESUMEN
Introducción: El proceso de decanulación consiste en la evaluación de las competencias de la vía aérea superior que conlleva al retiro definitivo de una cánula de traqueostomía. La predicción de su éxito resulta dificultosa debido al alto número de variables que la afectan. Para conseguir este objetivo, es fundamental contar con un protocolo adecuado a la institución de salud, que se sume a un manejo multidisciplinario.

Objetivo: El objetivo propuesto fue evaluar el éxito de decanulación en los pacientes adultos del Hospital Carlos van Buren que siguieron el protocolo institucional.

Material y método: Estudio de cohorte retrospectivo que se desarrolló durante el primer semestre del año 2016. Se evaluó el éxito de decanulación en pacientes adultos traqueostomizados que cumplieron con los criterios de inclusión. La muestra final fue de 18 pacientes, de los cuales se obtuvieron datos de interés desde las historias clínicas y registros del servicio de medicina y rehabilitación.

Resultados: De los 18 pacientes incluidos en el estudio ninguno cumplió con los criterios de ingreso al protocolo de decanulación institucional al momento de la evaluación inicial por el Servicio de Medicina Física y Rehabilitación, requiriendo todos entrenamiento para aplicar dicho protocolo. Finalmente, un total de 15 pacientes lograron decanulación exitosa, requiriendo una mediana de 22 días de entrenamiento fonoaudiológico y kinesiológico.

Conclusiones: Tras el entrenamiento terapéutico se logró 83\% de éxito en la decanulación, lo que requirió un trabajo coordinado entre las diferentes disciplinas del servicio de medicina física y rehabilitación y del apoyo del servicio de otorrinolaringología. Se deberá revaluar el protocolo institucional para ajustarlo a la realidad local.
\end{abstract}

Palabras clave: Traqueostomía, decanulación, rehabilitación.

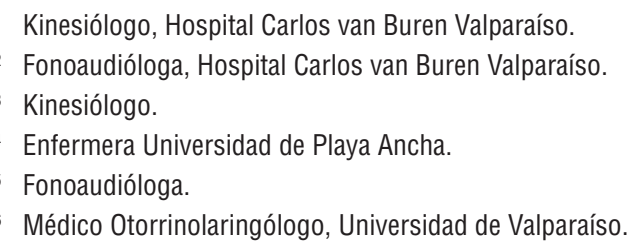




\section{ABSTRACT}

Introduction: The decannulation process consist in the evaluation of the upper airways competences which carry the definite extraction of the traqueostomy cannula. The prediction of its success is difficult due to the high number of variables that affect it. In order to reach this goal, is fundamental to count with an adequate protocol of the health institution, which assumes the multidisciplinary management.

Aim: The proposed goal was to evaluate the success of decannulation in adult patients of Carlos van Buren hospital who followed the institutional protocol.

Material and method: The cohort retrospective study that developed during the first semester of 2016. The decannulation success was evaluated in adult patients tracheostomized who fulfilled the inclusion criteria. The final sample was of 18 patients, of which were obtained data of interest from the clinical histories and register of the Medicine and Rehabilitation Service.

Results: Of the 18 patients in this study none of them met the entry criteria of the institutional decannulation protocol at the time of the initial evaluation by the Physical Medicine and Rehabilitation Service, requiring all training in order to apply the protocol. Finally, a total of 15 patients achieved a successful decannulation requiring a median of 22 days of phonoaudiological and kinesiological training.

Conclusions: After the therapeutic training it was achieved a 83\% of success in decannulation, which required a coordinated work between the different disciplines of the Physical Medicine and Rehabilitation Service and the support of the Otorhinolaryngology Service. The institutional protocol should be evaluated to adjust it to the local reality.

Key words: Tracheostomy, decannulation, rehabilitation.

\section{INTRODUCCIÓN}

El proceso de decanulación consiste en la evaluación de las competencias de vía aérea superior que conlleva al retiro definitivo de una cánula de traqueostomía $a^{1-4}$. Éste se lleva a cabo cuando el motivo por el cual fue indicado el procedimiento ha sido resuelto ${ }^{3,4}$. Las principales indicaciones de traqueostomía son: obstrucción de la vía aérea, ventilación mecánica prolongada, mal manejo de secreciones, entre otras ${ }^{5-7}$.

Dentro de los criterios para realizar la decanulación se encuentran destete exitoso del ventilador mecánico, estabilidad hemodinámica, evaluación nasofibroscópica normal o estenosis subglótica que comprometa menos del $30 \%$ del lumen aéreo, ausencia de delirio y/o trastornos psiquiátricos². Respecto a la deglución, ésta debe ser normal para saliva y/o disfagia leve, además contar con una adecuada fuerza de la musculatura respiratoria y un mecanismo tusígeno eficaz que permita un buen manejo de secreciones ${ }^{2,3}$.

La predicción del éxito en la decanulación en el usuario traqueostomizado es dificultosa debido al alto número de variables que lo afectan y a la heterogeneidad de éstas ${ }^{1,2,8-10}$. Para conseguir este objetivo es fundamental contar con un protocolo que se adecúe al ámbito de cada institución de salud y que refleje además su recurso humano, insumos y las características de los usuarios atendidos ${ }^{11}$.

Es por esto, que en el año 2016 el Servicio de Medicina Física y Rehabilitación en coordinación con el Servicio de Otorrinolaringología elaboró un protocolo de decanulación institucional para los usuarios adultos hospitalizados en el Hospital Carlos van Buren con el fin de asegurar la calidad de la atención y minimizar riesgos asociados (Figura 1).

Este protocolo resultó útil para aquellos usuarios que cumplieron con los requisitos para la decanulación en unidades de paciente crítico (UPC). Sin embargo, existió un grupo de pacientes que no cumplió con los criterios básicos de ingreso al protocolo o lo consiguieron hasta bien avanzada su hospitalización en unidades de menor complejidad. Estos usuarios se caracterizaron por tener un diagnóstico médico neurológico y/o complicaciones asociadas a estadías prolongadas en UPC, que 


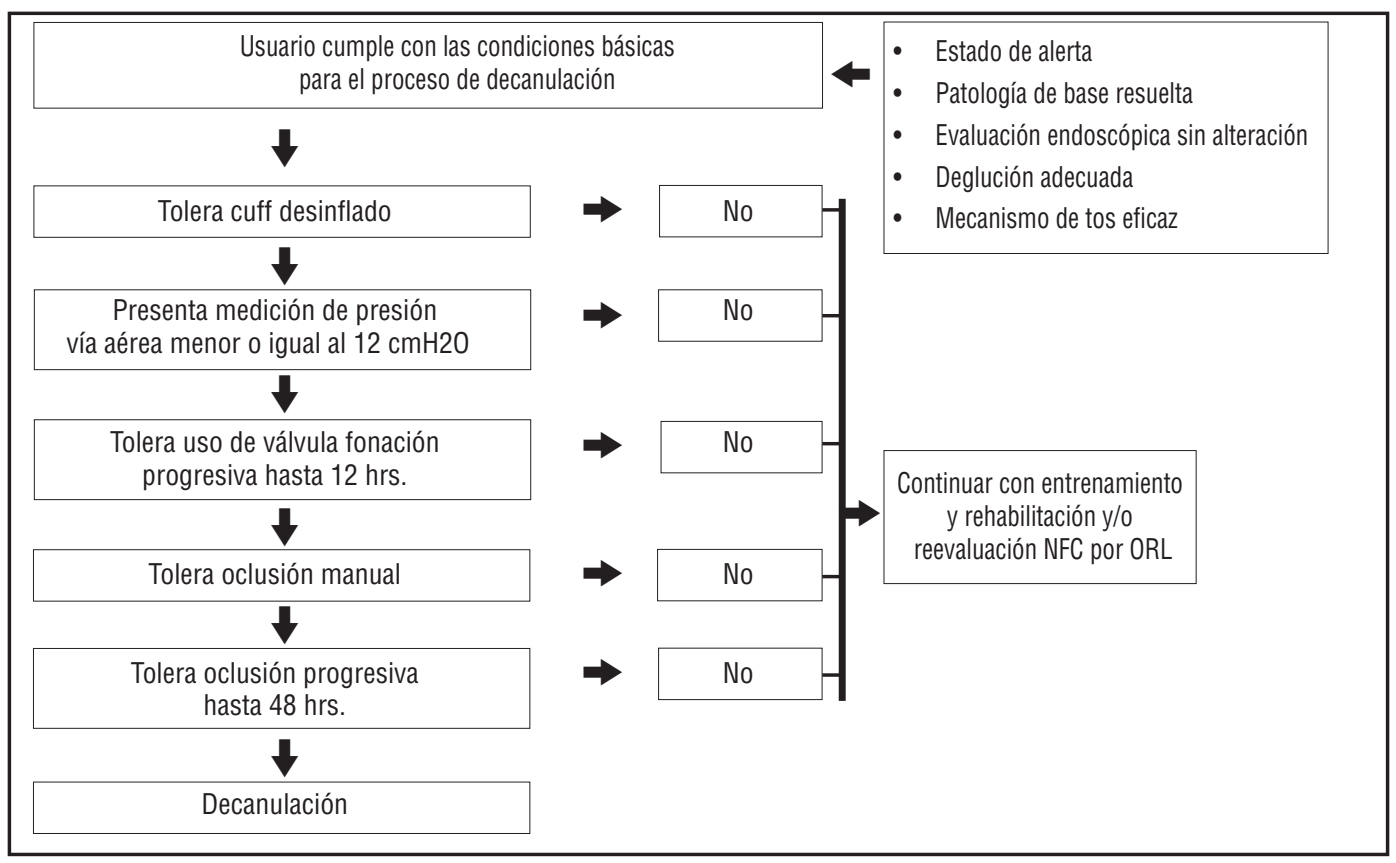

Figura 1. Flujograma protocolo de decanulación Hospital Carlos van Buren.

además requirieron de altos cuidados médicos y de enfermería ${ }^{10,12,13}$.

Es así, como estos usuarios fueron abordados con un enfoque multidisciplinario por el programa de neurorrehabilitación para cumplir con los criterios de ingreso al protocolo institucional y ser potencialmente decanulados. Finalmente, se ha planteado evaluar cuál es el éxito de decanulación en los pacientes pertenecientes al programa de neurorrehabilitación a los que se les aplicó dicho protocolo.

\section{MATERIAL Y MÉTODO}

Se realizó un estudio de cohorte retrospectivo en el que se incluyó a todos los pacientes traqueostomizados, ingresados al Programa de Neurorrehabilitación del Hospital Carlos van Buren de Valparaíso, provenientes de las unidades de neurocirugía, mediana complejidad médica y quirúrgica, hospitalizados durante los meses de enero a junio del año 2016.

Se definieron como criterios de inclusión para este estudio, por un lado, pacientes que hayan cursado toda su estadía hospitalaria en el Hospital Carlos van Buren. Por otro, desde el aspecto clínico; presentar estabilidad hemodinámica, procedimientos quirúrgicos resueltos y que la indicación de uso de traqueostomía no haya sido definitiva.

La obtención de datos fue realizada durante el mes de marzo del año 2017 desde la historia clínica de cada usuario, de manera anónima se registraron datos que consideraron: diagnóstico médico, tiempo de estadía hospitalaria, evaluaciones por otorrinolaringólogo, presión de la vía aérea previa a la decanulación, tiempo de traqueostomía previa al ingreso del programa de rehabilitación, tiempo de entrenamiento terapéutico y vía de alimentación al ingreso del programa. El estudio contó con la aprobación del Comité de Ético-Científico del Servicio de Salud Valparaíso-San Antonio, resolución ORD 230/2017.

En cuanto al tamaño de la muestra, según registros del programa de neurorrehabilitación, 38 pacientes traqueostomizados fueron atendidos en el periodo establecido, de los cuales 17 fueron trasladados a su hospital de origen y 3 requirieron de uso permanente de cánula de traqueostomía, por lo que no fueron considerados para el estudio, 
quedando finalmente una muestra de 18 pacientes que cumplían los criterios de inclusión.

Una vez que se consolidaron los datos extraídos de las historias clínicas, se realizó un análisis, donde se utilizó estadística no paramétrica. Los datos fueron descritos mediante frecuencias y porcentajes para las variables cualitativas y mediante medianas y rangos intercuartílicos para las variables numéricas.

El análisis del éxito de la decanulación consideró dos etapas: la primera, fue la aplicación del protocolo a través de una evaluación inicial y la segunda consideró una intervención coordinada entre las disciplinas de fonoaudiología y kinesiología, a través de un entrenamiento terapéutico. Además, se buscó determinar la relación entre diagnóstico médico y la severidad de la disfagia con el éxito de la decanulación, para lo cual se utilizó la prueba exacta de Fisher. Para determinar la asociación entre presión de vía aérea y el éxito de decanulación, y el diagnóstico médico con el tiempo de uso de traqueostomía se utilizó la prueba de Mann Whitney. A su vez, para determinar la correlación entre presión de vía aérea y el tiempo de uso de la traqueostomía se utilizó la prueba de correlación de Pearson. El análisis estadístico fue realizado en el software STATA 12, considerando siempre un nivel de significancia de $5 \%$.

\section{RESULTADOS}

De los 18 pacientes que cumplieron con los criterios de inclusión, 7 correspondieron a mujeres y 11 a hombres. La mediana de edad fue de 53,5 años. En cuanto al diagnóstico de ingreso hospitalario, 14 presentaban una patología neurológica. La mediana de la duración de estadía hospitalaria fue de 62,5 días (Tabla 1).

Todos los pacientes se alimentaban por sonda nasogástrica u orogástrica al ingreso del programa de neurorrehabilitación. El total de ellos también presentaba trastorno deglutorio, 13 de los cuales correspondía a disfagia severa. Al finalizar el proceso de decanulación, 16 pacientes lograron restituir la vía de alimentación oral, mientras que 2 sujetos requirieron de gastrostomía (Tabla 2).

De los pacientes evaluados ninguno cumplió con los criterios de ingreso al protocolo de decanulacion institucional. La mediana de tiempo de uso de la traqueostomía previo al ingreso del programa neurorrehabilitación fue de 16 días. Un total de 15 pacientes lograron exitosamente el proceso de decanulación, requiriendo una mediana de 22 días de entrenamiento terapéutico. Cada usuario fue evaluado entre 1 y 3 veces por el equipo de otorrinolaringología (Tabla 3).

Tabla 1. Características generales de pacientes adultos traqueostomizados, pertenecientes al programa de neurorehabilitación

\begin{tabular}{|ll|}
\hline Características & Valor \\
\hline Género, $n(\%)$ & \\
Femenino & $7(38,9)$ \\
Masculino & $11(61,1)$ \\
\hline Edad, mediana (RIC) años & $53,5(35-64)$ \\
Mínima & 15 \\
Máxima & 71 \\
\hline Estadía hospitalaria, mediana (RIC) días & $62,5(56-76)$ \\
\hline Causa hospitalaria, $n$ (\%) & \\
Patología respiratoria & $4(22,2)$ \\
Patología neurológica & $14(77,8)$ \\
\hline
\end{tabular}

RIC: rango intercuartílico. 
Tabla 2. Características de la disfagia de pacientes adultos traqueostomizados, pertenecientes al programa de neurorrehabilitación

\begin{tabular}{|lrl|}
\hline Características & Frecuencia (\%) \\
\hline Vía de alimentación al ingreso al programa de rehabilitación & 0 & $(0)$ \\
Oral & $18(100)$ \\
SNG/SOG & $0 \quad(0)$ \\
GTT & & \\
\hline Vía de alimentación al finalizar el proceso decanulación & 0 & $(0)$ \\
Sin disfagia & $16 \quad(88,9)$ \\
Oral & 0 & $(0)$ \\
SNG/SOG & $2 \quad(11,1)$ \\
GTT & & \\
\hline Severidad de la disfagia al ingreso al programa & 0 & $(0)$ \\
Sin disfagia & 1 & $(5,6)$ \\
Leve & 4 & $(22,2)$ \\
Moderada & $13 \quad(72,2)$ \\
Severa & & \\
\hline Severidad de la disfagia al finalizar el proceso decanulación & $11 \quad(61,1)$ \\
Sin disfagia & 5 & $(27,8)$ \\
Leve & 0 & $(0)$ \\
Moderada & $2(11,1)$ \\
Severa & 2 & \\
\hline
\end{tabular}

SNG: sonda nasogástrica, SOG: sonda orogástrica, GTT: gastrostomía.

Tabla 3. Características del proceso de decanulación de los pacientes adultos traqueostomizados, pertenecientes al programa de neurorrehabilitación

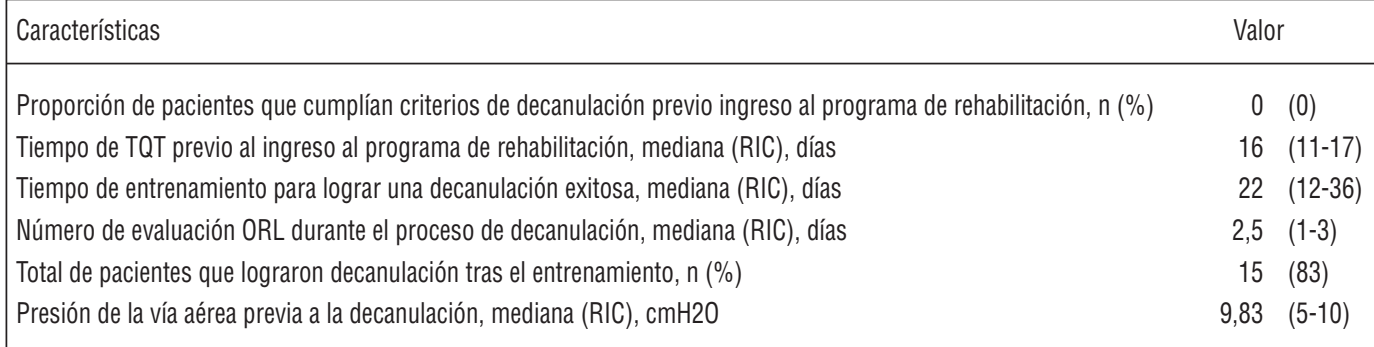

TQT: traqueostomía, RIC: rango intercuartílico.

En cuanto al tiempo de uso de traqueostomía según el diagnóstico de ingreso no se encontraron diferencias entre la patología neurológica y respiratoria (Prueba de Mann Whitney, $p=0,60$ ). Sin embargo, existió una mayor variabilidad en el grupo con patología neurológica (Figura 2).

Respecto a la mediana de presión de la vía aérea previo a la decanulación fue de 9,83 cmH20, encontrando una correlación positiva baja respecto al tiempo de uso de la cánula de traqueostomía (prueba correlación de Pearson 0,24) (Figura 3).

\section{DISCUSIÓN}

Dentro de las investigaciones sobre traqueostomía existen diferentes protocolos de decanulación, sin embargo, aún no se evidencia unificación 
de estos mismos. La elección entre las diversas técnicas de decanulación depende de cada institución y se relaciona con las necesidades de cada usuario. Por lo anterior, cabe destacar la importancia de elaborar protocolos universalmente aceptados para estandarizar los cuidados de los usuarios con traqueostomía $a^{8,14}$.

El éxito de la decanulación en este estudio fue de $83 \%$ con una mediana de 22 días, en comparación a lo descrito por Scrigna y cols $(2013)^{9}$ quienes obtuvieron $44,2 \%$ dentro de una mediana de 20 días. Por otro lado, Mackiewicz-Nartowicz y cols. $(2008)^{15}$ estudiaron pacientes neurológicos, en el cual consiguieron $31 \%$ de pacientes decanulados, con un amplio rango desde 1 a 389 semanas. En comparación con otras investigaciones, se han registrado hallazgos sobre los días promedio de decanulación variando entre 28 a $33^{13,16}$.

Uno de los factores más recurrentes que limitó la decanulación fue la disfagia severa con $72 \%$, por lo que inicialmente, el protocolo aplicado en este estudio no se ajustó a este grupo de usuarios. Por lo tanto, se requirió de terapia fonoaudiológica y kinesiológica previo al ingreso al protocolo institucional para cumplir con dicho criterio de inclusión. En este caso, se utilizaron estrategias terapéuticas como el uso de flujo translaríngeo, válvula de fonación, entrenamiento de musculatura respiratoria, ejercicios vocales, entrenamiento deglutorio, entre otros. Sin embargo, resulta fundamental estandarizar estas intervenciones, protocolizar y fomentar la investigación sobre su efectividad.

Es así, como la rehabilitación cobra relevancia, ya que, autores como Denison (2004) ${ }^{17}$ mencionan que no debemos condenar a los pacientes con trastorno deglutorio severo a una cánula de traqueostomía permanente. Del mismo modo y en complemento con el estudio de Frank y cols. $(2007)^{13}$ muestran que el entrenamiento de la deglución y la tos pueden conducir a una decanulación rápida y segura en pacientes traqueostomizados.

En cuanto a la medición de presión de vía aérea, nuestro promedio fue de $10 \mathrm{cmH}_{2} 0$ para los

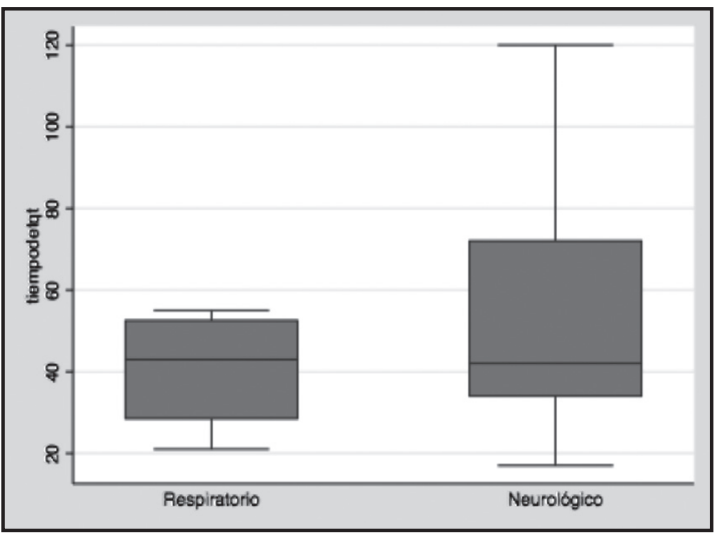

Figura 2. Asociación entre diagnóstico médico y tiempo de uso de cánula de la traqueostomía.

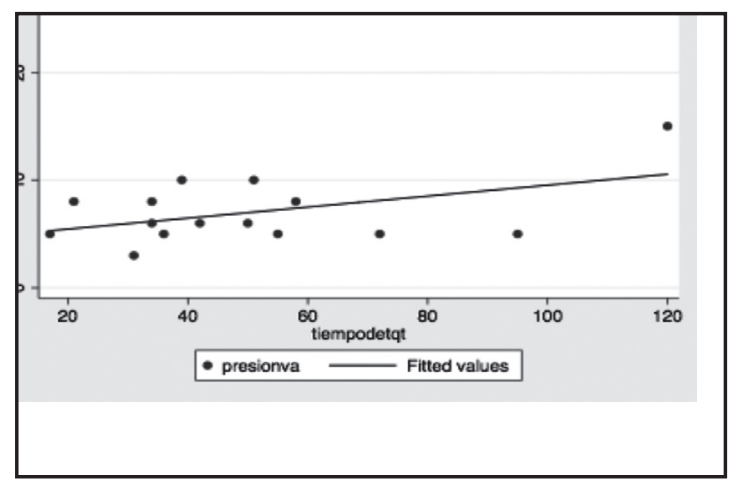

Figura 3. Correlación entre la presión de vía aérea y tiempo de uso de cánula de la traqueostomía. $R=0,24$, prueba de correlación de Pearson.

pacientes que se decanularon, por lo que valores más bajos fueron sugerentes de buen pronóstico en la decanulación en relación al tiempo. Este parámetro ha sido utilizado como un indicador indirecto de la permeabilidad de las vías respiratorias por encima de la traqueostomía ${ }^{18}$. Además, nuestros resultados concuerdan con los valores descritos por Johnson (2009) $)^{19}$, quien describe que valores bajo $10 \mathrm{CmH}_{2} \mathrm{O}$ orientan en el uso de estrategias terapéuticas tales como la válvula de fonación, oclusión y cambios de tubos de traqueostomía.

El tiempo de latencia para el ingreso al programa de neurorrehabilitación fueron 16 días en promedio, posterior a la instalación de la traqueostomía. Se ha descrito que la terapia temprana, de alta intensidad y multidisciplinaria reduce los tiempos de uso de traqueostomía durante la fase 
aguda de la hospitalización ${ }^{20}$. Además, otros estudios confirman que la implementación de un servicio dedicado a este tipo de pacientes desde la UPC, podría reducir los tiempos de decanulación y las tasas de complicaciones ${ }^{21}$. Según lo anterior, se deduce que un ingreso precoz al programa de neurorrehabilitación podría disminuir el tiempo de uso de traqueostomía, lo que resultaría interesante como tema para futuras investigaciones.

En este estudio, las atenciones de otorrinolaringología fueron en promedio 2,5, siendo su evaluación relevante para determinar aspectos como la indemnidad de la vía aérea y deglución previo a la decanulación ${ }^{22}$. Respecto a lo anterior, existe información controversial en cuanto a la utilización de la nasofibroscopía en la evaluación de la deglución previa a la decanulación, sin embargo, se considera como una alternativa segura en comparación con la decanulación tradicional ${ }^{8}$. En tanto, la evaluación nasofibroscópica tiene la ventaja de visualizar objetivamente la capacidad del paciente para manejar las secreciones directamente y permite tomar decisiones de forma rápida, segura y objetiva para guiar la decanulación ${ }^{23}$.

Por último, una gran falencia de este análisis es que no se encontraron registros sistemáticos

\section{BIBLIOGRAFÍA}

1. Alvarenga S, Berretin G, Silva M. Cânulas traqueais e disfagia orofaríngea: Uma revisão integrativa da literatura. Rev Bras Cir 2015; 44: 187-93.

2. Christopher K. Tracheostomy Decannulation. Respiratory Care 2005; 50: 538-41.

3. Maruvala S, Chandrashekhar R, Rajput R. Tracheostomy Decannulation: When and How? Research in Otolaryngology 2015; 4: 1-6.

4. Díaz P, Villalba D, Andreu M, Escobar M, Morel G, Lebus J, Rositi E. Decanular. Factores predictores de dificultad para la decanulación: Estudio de cohorte multicéntrico. Revista Americana de Medicina Respiratoria 2017; 17: 12-24.

5. De Leyn P, Bedert L, Delcroix M, Depuydt P, Lauwers G, SokoLov Y. Tracheotomy: clinical review and guidelines. European Journal of Cardio-Thoracic Surgery 2007; 32: 412-21. de la presión inspiratoria máxima, factor relevante en la valoración de la tos, ya que se considera un elemento determinante para el éxito de la decanulación ${ }^{24}$. Además, lo anterior toma mayor relevancia al considerar que en algunas patologías neurológicas se ha evidenciado disminución de la fuerza y resistencia de la musculatura respiratoria ${ }^{25}$. Así también, se ha documentado una rápida atrofia muscular diafragmática posterior a ventilación mecánica prolongada ${ }^{26}$.

\section{CONCLUSIÓN}

En este estudio se logró $83 \%$ de éxito en la decanulación, a través del entrenamiento terapéutico y el trabajo coordinado entre fonoaudiología, kinesiología y otorrinolaringología. Una propuesta multidisciplinaria y especializada en el área, favorece la toma de decisiones sobre el manejo de la traqueostomía. Además, facilita el desarrollo de directrices institucionales basada en la evidencia, por lo que, resulta fácil implementar y mejorar significativamente las tasas de decanulación y tolerancia de la alimentación oral27,28.

6. Cochrane L, Bailey C. Surgical aspects of tracheostomy in children. Paediatric Respiratory Reviews 2006; 7: 169-74.

7. Workman J, Bratton S. Pediatric Tracheostomy: perhaps good things come to those who don't wait. Minerva Anestesiol 2017; 83: 787-9.

8. Singh R, Saran S, Baronia A. The practice of tracheostomy decannulation: a systematic review. Journal of Intensive Care 2017; 5: 38. doi: 10.1186/s40560-017-0234-z.

9. Scrigna M, Plotnikow G, Feld V, Villalba D, Quiroga C, Leiva V, et al. Decanulación después de la estadía en UCI: Análisis de 181 pacientes traqueotomizados. Rev Amer Med Respiratoria 2013; 13: 58-63.

10. Mendes T, Cavalheiro L, Arevalo R, Sonegth R. Preliminary study on a preposal of an interdisciplinary flowchart of tracheostomy decannulation. Einstein 2008; 6: 1-6.

11. Villalba D, Lebus J, Quijano A, Bezz M, Plotnikow G. 
Retirada de la cánula de traqueostomía. Revisión Bibliográfica. Medicina Intensiva 2014; 31: 1-8.

12. Needham D. Mobilizing Patients in the Intensive Care Unit. JAMA 2008; 300: 1685-90.

13. Frank U, Mäder M, Sticher H. Dysphagic Patients with Tracheotomies: A Multidisciplinary Approach to Treatment and Decannulation Management. Dysphagia 2006; 22: 20-9.

14. Saavedra-Mendoza AG, Kaki-Caballero M. Puntos esenciales en el protocolo de decanulación traqueal. An Orl Mex 2014; 59: 254-61.

15. Mackiewicz-Nartowicz H, Mackiewicz-Milewska M, Lach S, Szymanska-Skrzypek A, Owczarek A, SInKIEWICZ A. Decannulation factors in patients after serious brain injuries. Advances in Palliative Medicine 2008; 7: 69-72.

16. Leung R, Campbell D, MacGregor L, Berkowitz R. Decannulation and Survival following Tracheostomy in an Intensive Care Unit. Annals of Otology, Rhinology \& Laryngology 2003; 112: 853-8.

17. Denison S. Decannulation of patients with longterm tracheostomies. Nursing Times 2004, 100: 58-9.

18. Utrarachki J, Pongsasnongkul J, Preutthipan A, Chantarojanaski T. Measurement of EndExpiratory Pressure as an Indicator of Airway Patency above Tracheostomy in Children. J Med Assoc Thai 2005; 88: 928-33.

19. Johnson DC, CampbelL SL, RabKIn JD. Tracheostomy tube manometry: evaluation of speaking valves, capping and need for downsizing. Clin Respir J 2009; 3: 8-14.

20. Berney L, Wasserfallen J, Grant K, Levivier M, Simon C, FAouzI M, et AL. Acute neurorehabilitation: does a neurosensory and coordinated interdisciplinary programme reduce tracheostomy weaning time and weaning failure? Neurorehabilitation 2014; 34: 809-17.
21. Stelfox HT, Hess DR, Schmidt UH. A North American Survey of Respiratory Therapist and Physician Tracheostomy Decannulation Practices. Respiratory Care 2009; 54: 165864.

22. Alvo A, Ovalaría C. Decanulación y evaluación de la deglución del pacientetraqueotomizado en cuidados intensivos no-neurocríticos. Acta Otorrinolaringología Esp 2014; 65: 114-9.

23. Warnecke T, Suntrup S, Teismann I, Hamacher $C$, Oelenberg S, Dziewas R. Standardized Endoscopic Swallowing Evaluation for Tracheostomy Decannulation in Critically III Neurologic Patients. Critical Care Medicine 2013; 7: 1728-32.

24. Villalba D, Lebus J, Quijano A, Bezzi M, Plotnikow G. Retirada de la cánula de traqueostomía. Revisión Bibliográfica. Medicina Intensiva 2014; 31: 1-11.

25. Menezes K, Nascimento L, Ada L, Polese J, Avelino P, Teixeira-Salmela L. Respiratory muscle training increases respiratory muscle strength and reduces respiratory complications after stroke: a systematic review. Journal of Physiotherapy 2016; 62: 138-44.

26. Schepens T, Verbrugghe M, Dams K, Corthouts $B$, Parizel $P$, Jorens $P$. The course of diaphragm atrophy in ventilated patients assessed with ultrasound: a longitudinal cohort study. Schepens et al. Critical Care 2015; 19: 422.

27. De Mestral C. Impact of a specialized multidisciplinary tracheostomy team on tracheostomy care in critically ill patients. Canadian Journal of Surgery 2011; 54: 167-72.

28. Mah J, Staff I, Fisher S, Butler K. Improving Decannulation and Swallowing Function: A Comprehensive, Multidisciplinary Approach to Post-Tracheostomy Care. Respiratory Care 2017; 62: 137-43.

Dirección: Cristian Cortés Quinchavil

Avenida Las Lomas 331, Valparaíso

E mail: c.cortes.q@gmail.com 